\title{
Decreased cortical glucose metabolism correlates with hippocampal atrophy in Alzheimer's disease as shown by MRI and PET
}

Satoshi Yamaguchi, Kenichi Meguro, Masatoshi Itoh, Chika Hayasaka, Masumi Shimada, Hideki Yamazaki, Atsushi Yamadori

\begin{abstract}
Objective-To investigate the relation between atrophy of the hippocampus and parahippocampal gyrus (the \% hippocampal area) and cerebral metabolic rate for glucose (CMRGlc) in Alzheimer's disease.
\end{abstract}

Methods-13 patients with probable Alzheimer's disease by NINCDS-ADRDA criteria (six men; seven women, mean age 71 years, mini mental state $13 \cdot 8$ (SD $4 \cdot 6$ )) and age matched controls were studied. T1 weighted MRI (0.5T) images were used for evaluation of the hippocampal area. With a digitiser system, a percentage of the hippocampal area to the brain (the \% hippocampal area) was calculated. Eight patients received another $T 1$ weighted MRI (1.5T) for further evaluation of the minimum thickness of the hippocampus. Regional CMRGlc (rCMRGlc) was measured using PET and the FDG technique. Results-The hippocampal area in patients with Alzheimer's disease was significantly lower than that of controls $(P<0 \cdot 01)$. All the cortical rCMRGlc values in patients with Alzheimer's disease were lower than those of controls $(\mathbf{P}<\mathbf{0 . 0 1})$. A significant correlation $(P<0.05)$ was found between the \% hippocampal area and rCMRGlc in the temporal lobe, temporoparieto-occipital (TPO) region, and frontal lobe in Alzheimer's disease. There was a significant correlation between minimal hippocampal thickness and ipsilateral TPO metabolism on both sides.

Conclusion-The ipsilateral correlation between hippocampal atrophy and decreased TPO metabolism in Alzheimer's disease suggests a functional relation and the asymmetries show that Alzheimer's disease is an asymmetric disease in its early stages.

(F Neurol Neurosurg Psychiatry 1997;62:596-600)

Keywords: Alzheimer's disease; hippocampus; cerebral metabolic rate for glucose

Neuroimaging studies on Alzheimer's disease have been performed using CT or MRI for morphology and single photon emission CT (SPECT) or PET for function. Previous morphological studies have shown that: (1) patients with Alzheimer's disease have greater brain atrophy than aged normal subjects, ${ }^{1-3}$ including cortical atrophy and ventricular enlargement; (2) the temporal lobe, especially the hippocampus, shows atrophy in Alzheimer's disease..$^{4-9}$ Functional studies (circulation and metabolism) have shown that: (1) cerebral blood flow (CBF) and cerebral metabolic rate for oxygen $\left(\mathrm{CMRO}_{2}\right)$ are lower in Alzheimer's disease than controls, especially in the temporoparieto-occipital (TPO) region $^{10-12}$; (2) CMRGlc in Alzheimer's disease is also decreased in the temporal lobe and TPO region, and decreased CMRGlc in the right and left TPO regions is associated with impaired visuospatial and verbal functions as shown by the Wechsler adult intelligence scale (WAIS). ${ }^{13-17}$

In view of the relation between morphology and function, Jobst $e t$ al ${ }^{18}$ studied the relation between atrophy of the hippocampus shown by CT and CBF by SPECT in Alzheimer's disease. They found an association between hippocampal atrophy and ipsilateral temporoparietal hypoperfusion. However, there has been no study of the relation between atrophy of the hippocampus and CMRGlc, although both were found to be important in Alzheimer's disease. In the present study, we tried to confirm the findings of Jobst et al by using PET measurements of glucose consumption in Alzheimer's disease.

\section{Subjects and methods}

SUBJECTS

Ten normal elderly subjects (five men and five women, ages $68-78$ years, mean age 73 years) and 13 patients with Alzheimer's disease (six men and seven women, ages 56-88 years, mean age 71 years) were studied. There was no statistical difference in mean age between the groups. The normal subjects underwent medical interview, physical and neurological examinations, laboratory tests, ECG, and CT. They displayed no cognitive impairment based on clinical observation, no risk factors for cerebrovascular disease such as hypertension (except for smoking histories), and no history of head injury or any other disorders that could affect brain function. The brain CT findings were normal.

The diagnosis of Alzheimer's disease was based on the NINCDS-ADRDA criteria for probable Alzheimer's disease. ${ }^{19}$ Brain MRI (described below) in Alzheimer's disease disclosed brain atrophy without any infarctions. All the patients with Alzheimer's disease were studied within five years of onset of symptoms and showed mild to moderate decline of intelli- 
Table 1 Clinical characteristics of the patients with Alzheimer's disease

\begin{tabular}{|c|c|c|c|c|c|c|c|c|}
\hline \multirow{2}{*}{$\begin{array}{l}\text { Patient } \\
\text { No }\end{array}$} & \multirow{2}{*}{$\begin{array}{l}\text { Age } \\
(y)\end{array}$} & \multirow[b]{2}{*}{ Sex } & \multirow{2}{*}{$\begin{array}{l}\text { Duration } \\
\text { (y) }\end{array}$} & \multirow[b]{2}{*}{$M M S$} & \multirow[b]{2}{*}{$T K-I Q$} & \multicolumn{3}{|c|}{ WAIS-R subscore } \\
\hline & & & & & & $V I Q$ & $P I Q$ & $T I Q$ \\
\hline 1 & 88 & F & 3 & 10 & 20 & 12 & 0 & 12 \\
\hline 2 & 74 & $\mathrm{~F}$ & 2 & 17 & 52 & 27 & 30 & 57 \\
\hline 3 & 58 & $\mathbf{M}$ & 3 & 9 & 19 & 15 & 0 & 15 \\
\hline 4 & 77 & $\mathbf{M}$ & 5 & 9 & 18 & 0 & 8 & 8 \\
\hline 5 & 60 & $\mathrm{~F}$ & 2 & 16 & 62 & 33 & 13 & 46 \\
\hline 6 & 77 & $F$ & 3 & 22 & 55 & 57 & 35 & 92 \\
\hline 7 & 82 & $\mathbf{M}$ & 3 & 15 & 36 & 27 & 3 & 30 \\
\hline 8 & 80 & $F$ & 2 & 16 & 43 & 45 & 8 & 53 \\
\hline 9 & 74 & $\mathrm{~F}$ & 5 & 10 & 34 & 18 & 10 & 28 \\
\hline 10 & 79 & $\mathbf{M}$ & 5 & 21 & 45 & 54 & 20 & 74 \\
\hline 11 & 59 & $\mathrm{~F}$ & 2 & 14 & 40 & 63 & 0 & 63 \\
\hline 12 & 64 & $\mathbf{M}$ & 5 & 12 & 40 & 39 & 13 & 52 \\
\hline 13 & 56 & $\mathbf{M}$ & 2 & 8 & 54 & 18 & 8 & 6 \\
\hline
\end{tabular}

MMS = mini mental state; WAIS-R = Wechsler adult intelligence scale-revised; VIQ = verba IQ; $P I Q=$ performance IQ; TIQ = total $I Q ; T K=$ Tanaka-Binet test.

gence (mini mental state (MMS) ${ }^{20}$ range, $8-22$, mean (SD) $13.8(4 \cdot 6)$ ). They did not receive any medication which might affect $\mathrm{CBF}$ and metabolism. All received WAIS-R (revised) ${ }^{21}$ and Tanaka-Binet tests. ${ }^{22}$ Table 1 gives the clinical characteristics of the patients with Alzheimer's disease.

This study was approved by the medical ethics committee of the Cyclotron Radioisotope Center at Tohoku University, and informed consent was received from all the subjects and their families.

\section{MAGNETIC RESONANCE IMAGING}

The MRI was carried out with a $0.5 \mathrm{~T}$ MRVectra (GE-YMS, Japan). T1 weighted images (TR/TE 300/15) were used for evaluation of atrophy of the hippocampus and parahippocampal gyrus, which we defined as the \% hippocampal area. The hippocampus was identified by the sagittal slices, then a semiaxial slice parallel to the hippocampus was

Figure 1 MRI measurement of the hippocampal area and the temporal lobe. A sagittal slice through the midpoint of the eye is obtained, and a slice parallel to the hippocampus is scanned; then planes perpendicular to the plane parallel to the hippocampus are obtained (the semicoronal planes). The $T 1$ weighted images of this slice are input into the computer system which enlarged them about twofold, and using a digitiser system, the hippocampus and the parahippocampal gyrus (the hippocampal area) is traced manually.

Similarly, the temporal area is measured using the same plane. The percentage of the hippocampal area to the brain area (the \% hippocampal area) and the temporal area to the brain area (the \% temporal lobe) are calculated.
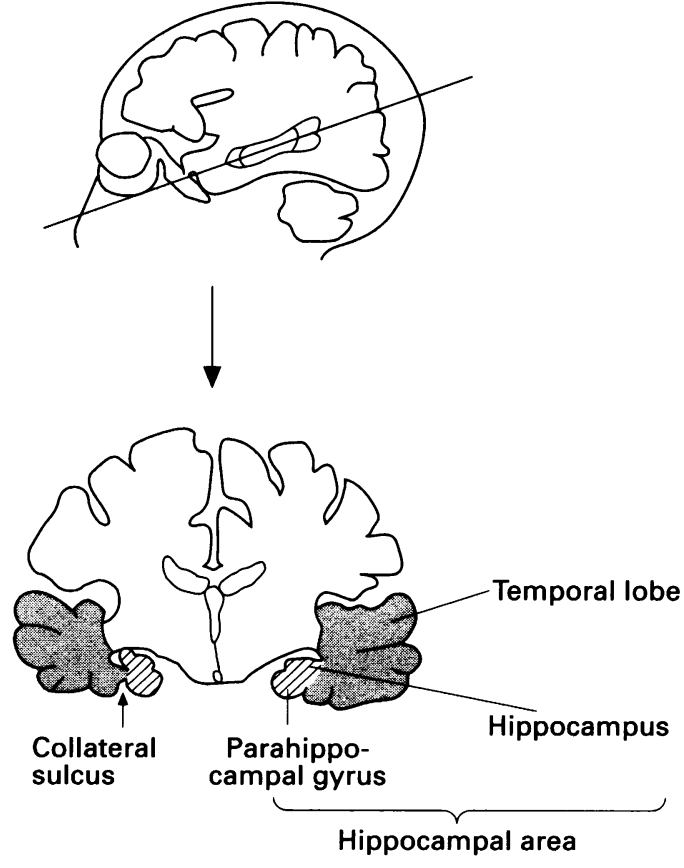

$$
\begin{aligned}
\text { Temporal lobe }(\%) & =\frac{T L(b / t)}{B \text { rain }} \times 100 \\
\text { Hippocampal area }(\%) & =\frac{H A(b / t)}{B \text { Bain }} \times 100
\end{aligned}
$$

scanned. Finally, planes perpendicular to the semiaxial planes were obtained (the semicoronal planes).

The $\mathrm{T} 1$ weighted image of the semicoronal plane which had the largest area of the hippocampus was transferred to a computer system which enlarged the image about twofold, and the hippocampal area was traced manually using a digitiser system. Figure 1 shows this process. Similarly, the temporal area was measured from the same semicoronal plane. Using routine orbitomeatal axial planes $+30,+50$, and $+70 \mathrm{~mm}$, the brain area and the area of the skull cavity were also evaluated.

The percentage of the hippocampal area to the brain area (the hippocampal area), the temporal area to the brain area (the temporal area), and the brain area to the skull cavity (the brain area) were calculated. The measurements were performed by two neuroradiologists who did not know the PET findings: each neuroradiologist made two measurements and the average values of the four measurements were used. The average values of the bilateral hippocampal area and the \% temporal area were calculated and used for analysis as it was difficult to show a left-right difference with this system. The within and between reader reproducibility of all the MRI variables were calculated as follows:

1 - (value 1 - value 2$) /($ value $1+$ value 2$) / 2$

For the within reader reproducibility, values 1 and 2 were assessed by two radiologists. For the between reader reproducibility, values 1 and 2 were assessed by the same radiologist. Both reproducibilities were better than 0.95.

An axial T1 weighted MRI parallel to the orbitomeatal line was examined at the same head position as in the PET study to obtain accurate overlaps (described below), and an axial T2 weighted (TR/TE 2000/100) MRI was performed to exclude infarctions.

\section{Additional MRI}

To show left-right differences, eight patients in the Alzheimer's disease group received another T1 weighted MRI (1.5T, Magnetom, Siemens, FRG) for further evaluation of the hippocampus as the digitiser system of $0.5 \mathrm{~T} \mathrm{MRI} \mathrm{could}$ not detect small differences. Using a new semiaxial plane, the minimum thickness of the hippocampus was measured and divided by the brain width of the same plane ${ }^{\text {? }}$.

POSITRON EMISSION TOMOGRAPHY

A PET study was performed with a model PT931 scanner (CTI Inc, USA: axial/transaxial resolutions; $8 \mathrm{~mm}$ ), according to the FDG method. ${ }^{2324}$ A short cannula was placed in a radial artery for blood sampling. Each subject was positioned in the scanner, with the orbitomeatal line parallel to the detector rings according to the brain slices by MRI. A cross of light was projected on to marks on the subject's head from three dimensions, and the heads were set at the standard points of 30 and $77 \mathrm{~mm}$ above and parallel to the orbitomeatal line. All studies were conducted in a quiet, semidarkened room. The subjects' eyes were open and their ears were not plugged. 
Table 2 Cerebral metabolic rate for glucose for (rCMRGIc) the two groups

\begin{tabular}{|c|c|c|}
\hline \multirow[b]{2}{*}{ Brain regions } & \multicolumn{2}{|l|}{ rCMRGIc } \\
\hline & Normal $(n=10)$ & $A D(n=13)$ \\
\hline Mean & $6 \cdot 81(1.25)$ & $4.50(0.52)$ \\
\hline Upper frontal & $8.35(1.86)$ & $5 \cdot 18(1 \cdot 11)$ \\
\hline Anterior frontal & $7 \cdot 42(1.80)$ & $4.28(0.63)$ \\
\hline Inferior frontal & $6.42(1.48)$ & $4.62(0.90)$ \\
\hline Parietal & $8.03(1.95)$ & $4 \cdot 71(1.06)$ \\
\hline TPO & $6 \cdot 87(1.44)$ & $3.74(0.45)$ \\
\hline Primary auditory & $8.57(1.81)$ & $4.76(0.68)$ \\
\hline Temporal & $6.42(1.48)$ & $4.31(0.93)$ \\
\hline Hippocampus & $5.41(1.95)$ & $3.22(1.85)$ \\
\hline Primary visual & $8.88(1.75)$ & $5.04(0.80)$ \\
\hline Occipital & $7.43(1.48)$ & $4.06(0.57)$ \\
\hline Basal ganglia & $6.53(1.24)$ & $5.17(0.79)$ \\
\hline Cerebellum & $7 \cdot 31(1.55)$ & $5.04(0.14)$ \\
\hline White matter & $5.72(1.33)$ & $5.07(0.74)$ \\
\hline
\end{tabular}

A 20 minute transmission scan using a ${ }^{68} \mathrm{Ge} /{ }^{68} \mathrm{Ga}$ external ring source was performed. A total of $5 \mathrm{mCi}$ to $12 \mathrm{mCi}$ FDG were injected as an intravenous bolus. Thirty to 45 minutes after the injection, a series of two emission scans was performed. Each emission datum was simultaneously collected from seven contiguous axial sections. Fourteen slices parallel to the orbitomeatal line with a slice thickness of 6 $\mathrm{mm}$, encompassing virtually the whole brain, were analysed. Twenty blood samples were collected as follows: from the FDG injection to two minutes, every 20 seconds, then at $2 \cdot 5,3$, $4,5,7 \cdot 5,10,15,20,25,30,40,50$, and 60 minutes. The samples were immediately centrifuged, and the plasma radioactivities were measured with a cross calibrated well counter. The plasma glucose concentrations were measured every 10 minutes.

\section{DATA ANALYSIS}

Two different pairs of axial MRI and PET images were roughly registered and matched with each other at the same brain slices. ${ }^{25}$ To ascertain the anatomical position of each brain structure, the position of regions of interest (round ROIs, $2.7 \mathrm{~cm}^{2}$ ) were manually defined using the overlapped images. A total of 13 ROIs in each hemisphere were placed on these images. Each ROI was positioned in the relevant region with reference to the anatomical atlases $^{2627}$ with no overlapping. The rCMRGlc was measured in the following bilateral regions: upper frontal, anterior frontal, inferior frontal, primary auditory, temporal, parietal, TPO, hippocampus, primary visual, occipital, basal ganglia, cerebellum, and white matter. The mean value of left and right cortical CMRGlcs in each ROI was calculated and used for analysis.

Figure 2 Correlation between the \% hippocampal area and mean cortical cerebral metabolic rate for glucose (CMRGlc) for the Alzheimer's disease group.

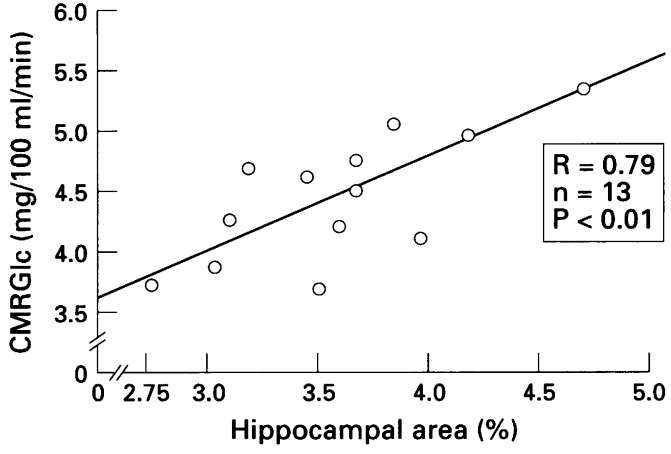

Statistical analysis was performed using Student's $t$ test, and Pearson's and Spearman's correlation coefficients.

\section{Additional analysis}

For the eight patients with Alzheimer's disease who received a $1.5 \mathrm{~T}$ MRI, a $\chi^{2}$ test was used to detect a relation between the hippocampal asymmetry and the CMRGlc asymmetry in all ROIs measured.

\section{Results}

The mean hippocampal areas of the normal group and the Alzheimer's disease group were 5.5 (SD 0.8$) \%$ and 3.4 (SD 1.4 ) \% respectively ( $t$ test, $\mathrm{P}<0.01$ ). The brain area and the temporal area of the patients with Alzheimer's disease were also significantly lower than those of the normal (data not shown).

Table 2 shows the rCMRGlc values of the two groups. All the rCMRGlc values except for that of the white matter of the Alzheimer's disease group were lower than those of the normal group ( $t$ test, $\mathrm{P}<0.01$ ).

The correlation between the brain area and the mean cortical CMRGlc of the Alzheimer's disease group was not significant; nor were the temporal area and the mean cortical CMRGlc. Figure 2 shows the correlation between the hippocampal area and the mean cortical CMRGlc of the Alzheimer's disease group; a significant correlation was found ( $P<0.05$, Pearson).

Figure 3 shows the relation between the hippocampal area and the rCMRGlc in the Alzheimer's disease group. The rCMRGlc values of the temporal lobe, the TPO region, and the frontal lobe were significantly correlated $(\mathrm{p}<0.05$, Pearson). For the rCMRGlc ratio to that of the mean cortical CMRGlc (rCMRGlc/CMRGlc), that of the TPO region was significantly correlated with the hippocampal area (Spearman rank correlation). There was no correlation with the other ROIs, such as the frontal lobe.

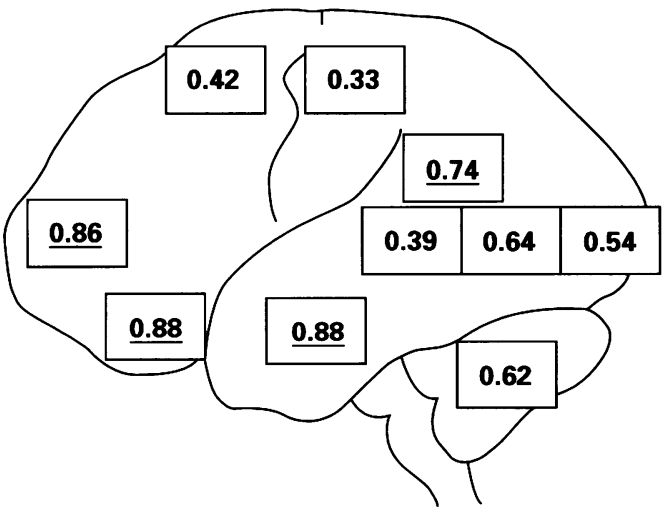

Figure 3 Correlation coefficients between the hippocampal area and cortical cerebral metabolic rate for glucose (CMRGlc). The relations between the hippocampal area and the regional CMRGlc (except for those of the basal ganglia, hippocampus, and white matter) in the Alzheimer's disease group are shown. The numbers in the boxes are Pearson's correlation coefficients in each area of the brain. Underlining indicates significant data: the CMRGlc values of the temporal lobe, the temporoparietooccipital region, and the frontal lobe are correlated $(P<0.05)$. 
ADDITIONAL ANALYSIS

In five of the eight patients with Alzheimer's disease, the minimum thickness of the left hippocampus was smaller than the right, and in the remaining three, the reverse pattern was present. For metabolic asymmetry, all three with smaller right hippocampi had right TPO metabolism lower than the left; and all five with smaller left hippocampi had left TPO metabolism lower than the right. A $2 \times 2 \chi^{2}$ test disclosed that left-right hippocampal asymmetry and metabolic asymmetry (right $<$ left or right $>$ left) were significantly correlated $\left(\chi^{2}>\chi_{1}^{2}(P<0.005)\right)$. There was no such relation for the other ROIs, such as the frontal lobe.

\section{Discussion}

In this study, we examined patients with Alzheimer's disease, using MRI and PET, and found that the hippocampal area was significantly correlated with the average value of cortical CMRGlc. For regional metabolism, those of the frontal lobe, the temporal lobe, and the TPO region were correlated. For rCMRGlc of the hippocampus, there were no significant correlations, probably because of a partial volume due to atrophy giving larger SDs to rCMRGlc values.

Regarding the MRI assessment, we performed tracing of the hippocampal area enlarged on a computer screen. Jack et $a l^{28}$ studied MRI based area measurements of the hippocampus and found that the tracing technique was more accurate, especially for the smaller cylinders, than the random marking technique, although the results of the method may have been affected by the observer's perception of object boundary, experience, knowledge of relevant anatomy, and complexity of the object boundary. ${ }^{28}$ The measurements in this study were taken by two radiologists (each made two observations), and the average values of four measurements was used. As the between and within reader reproducibilities were found to be better than 0.95 , we assume that the MRI data were reliable.

The pathological features of Alzheimer's disease, such as neuronal cell loss, neurofibrillary tangles, and senile plaques, are found in the hippocampal area and in the association neocortices. ${ }^{29-32}$ Hippocampal atrophy as shown by CT or MRI as well as decreased rCMRGlc in the temporal lobe, parietal lobe, or TPO region as shown by PET are well known neuroimaging findings. The fact that both were correlated indicates a possibility that the hippocampal area and the TPO region are "related". This speculation is supported by the result that the rCMRGlc/CMRGlc ratio of the TPO region was significantly correlated with the hippocampal area in Alzheimer's disease.

However, according to Haxby and Grady et $a l,{ }^{173334}$ Alzheimer's disease is an inherently asymmetric disease: metabolic reductions in the parietal association cortex and increased left-right metabolic asymmetry were found in mild to moderate Alzheimer's disease, and this metabolic asymmetry is evident before the neuropsychological consequences of that dysfunction are demonstrable. Jobst et al ${ }^{18}$ noted that hippocampal atrophy was accompanied by ipsilateral temporoparietal perfusion. Our additional data that morphological asymmetry of the hippocampus and a metabolic asymmetry of the TPO region was correlated, support the findings of others. ${ }^{1733} 34$ Further analysis with larger sample size is needed to determine the possible role of other regions such as the frontal lobe.

We are grateful to the staff of Miyama and Miki Hospitals, all the PET members at Tohoku University, and Drs $T$ Yamaguchi, H Matsui, H Sasaki, and H Fukuda. We also thank Drs JC Baron and C Chavoix for valuable comments.

1 Lang C, Herholz K, Huk W, Feistel H. Diagnostic differentiation of dementia disease by modern imaging proceentiation of dementia disease by modern imaging
dures. Fortschr Neurol Psychiatr 1990;58:380-98.

2 Erkinjunti T, Ketonen L, Sulkava R, Vuorialho M, Palo J. $\mathrm{CT}$ in the differential diagnosis between Alzheimer's disease and vascular dementia. Acta Neurol Scand 1987;75: $262-70$

3 Albert M, Naeser MA, Levine HL, Garvey AJ. Ventricular size in patients with presenile dementia of the Alzheimer's type. Arch Neurol 1984;41:1258-63.

4 deLeon MJ, George AE, Stylopoulos LA, et al. Early marker for Alzheimer's disease: the atrophic hippocampus. Lancet 1989;ii:672-3.

5 Seab JP, Jagust WJ, Wong STS, et al. Quantitative NMR measurements of hippocampal atrophy in Alzheimer's disease. Magn Reson Med 1988;8:200-8.

6 Kesslak JP, Nalciogiu O, Cotman CW. Quantification of magnetic resonance scan for hippocampal and parahippocampal atrophy in Alzheimer's disease. Neurology 991;41:51-4.

7 Jobst KA, Smith AD, Szatmari M, et al. Detection in life of confirmed Alzheimer's disease using a simple measurement of medial temporal lobe atrophy by computed tomography. Lancet 1992;340:1179-83.

8 Jack Jr CR, Pelerson RC, O'Brien PC, et al. MR-based hippocampal volumetry in the diagnosis of Alzheimer's disease. Neurology 1992;42:183-8.

9 Scheltens $\mathrm{Ph}$, Leys D, Barkhof $\mathrm{F}$, et al. Atrophy of medial temporal lobes on MRI in probable Alzheimer's disease and normal aging: diagnostic value and neuropsychological correlates. $\mathcal{f}$ Neurol Neorosurg Psychiatry 1992;55: 967-72.

10 Frackowiak RSJ, Pozzilli C, Legg NJ, et al. Regional cerebral oxygen supply and utilization in dementia. Brain 1981;104:753-78.

11 Komatani A, Yamaguchi K, Sugai Y, et al. Assessment of demented patients by dynamic SPECT of inhalated xenon-133. F Nucl Med 1988;29:1621-6.

12 DeKosky ST, Shih WJ, Schmitt FA, Coupal J, Kirkpatrick C. Assessing utility of single photon emission tomography (SPECT) scan in Alzheimer's disease: correlation with cognitive severity. Alzheimer Dis Assoc Disord 1990;4: 14-23.

13 Chase TN, Foster NL, Fedio P, Brooks R, Mansi L, Chiro GO. Regional cortical dysfunction in Alzheimer's disease as determined by positron emission tomography. Ann Neurol 1984;15(suppl):S170-4.

14 Koss E, Friedland RP, Ober BA, Jagust WJ. Difference in lateral hemispheric asymmetries of glucose utilization between early-and late-onset Alzheimer-type dementia. Am ₹ Psychiatry 1985;142:638-40.

15 Cutler NR, Haxby JV, Duara R, et al. Clinical history, brain metabolism, and neuropsychological function in metabolism, and neuropsychological function

16 Foster NL, Chase TN, Patronas NJ, Gillespie MM, Fedio P. Cerebral mapping of apraxia in Alzheimer's disease by positron emission tomography. Ann Neurol 1986;19. $139-43$

17 Rapoport SI, Horwitz B, Haxby JV, Grady CL. Alzheimer's disease: metabolic uncoupling of associative brain regions. Can $\mathcal{F}$ Neurol Sci 1986;13:540-5.

18 Jobst KA, Smith AD, Barker CS, et al. Association of atrophy of the medial temporal lobe with reduced blood flow in the posterior parietotemporal cortex in patients with in the posterior parietorith flinical and pathological diagnosis of Alzheim

19 Neurol Neorosurg Psychiatry 1992;55:190-4. nosis of Alzheimer's disease: report of the NINCDSADRDA work group under the auspices of department of health and human services task force on Alzheimer's dishealth and human services task for

20 Folstein MF, Folstein SE, McHugh PR. Mini-mental state: a practical method for grading the cognitive state of a practical method for grading the cognitive state of
patients for the clinician. $\Im$ Psychiatr Res $1975 ; 12: 189-98$

21 Wechsler adult intelligence scale-revised, Japanese version. Tokyo: The Psychological Co,1990.
sichsler adult intelligence scale-revised,

22 Tanaka-Binet test, Japanese version. Tokyo: Tanaka Institute for Education, 1987

23 Phelps ME, Huang SC, Hoffman EJ, Selin C, Sokoloff L, Kuhe DE. Tomographic measurement of local glucose 
metabolic rate in humans with (FO-18)2-fluoro-2-deoxyD-glucose: validation of method. Ann Neurol 1979;6: 371-88.

24 Reivich M, Kuhl D, Wolf AP, et al. The ${ }^{18} \mathrm{~F}$-fluorodeoxyglucose method for the measurement of local cerebral glucose utilization in man. Circ Res 1979;44:127-37.

25 Nagasawa H, Kogure K, Fujiwara T, Itoh M, Ido T. Metabolic disturbances in exo-focal brain areas after cortical stroke studied by positron emission tomography. $\mathscr{f}$ tical stroke studied by positron

26 Salamon G, Huang YP. Computed tomography of the brain. Berlin: Springer-Verlag, 1980

27 Talairach J, Tournoux P. Co-planar stereotaxic atlas of the human brain. 3-dimensional proportional system: an approach to cerebral imaging. Stuttgart: Georg Thieme Verlag, 1988.

28 Jack Jr CR, Bentley MD, Twomey CK, Zinsmeister AR. MR imaging-based volume measurements of the hippocampal formation and anterior temporal lobe: validation studies. Radiology 1990;176:205-9.
29 Ball MJ. Neuronal loss, neurofibrillary tangles and granulovacuolar degeneration in the hippocampus with aging and dementia-a quantitative study. Acta Neuropathol 1977;37:111-8.

30 Ball MJ, Lo P. Granulovacuolar degeneration in the aging brain and in dementia. $\mathcal{F}$ Neuropathol Exp Neurol 1977; 36:474-87.

31 Ball MJ. Topographic distribution of neurofibrillary tangles and granulovacuolar degeneration in hippocampal cortex of aging and demented patient. A quantitative study. Acta Neuropathol 1978;42:73-80.

32 Pandya DN, Seltzer B. Association areas of the cerebral cortex. Trends Neurosci 1982;5386-90.

33 Duara R, Grady CL, Haxby JV, et al. Positron emission tomography in Alzheimer's disease. Neurology 1986;36: 879-87.

34 Haxby JV, Grady CL, Duara R, Schlageter N, Berg G, Rapoport SI. Neocortical metabolic abnormalities precede nonmemory cognitive defects in early Alzheimer'stype dementia. Arch Neurol 1986;43:882-5. 